\title{
FULLY COMMUTATIVE KAZHDAN-LUSZTIG CELLS
}

\author{
R.M. Green And J. Losonczy \\ Department of Mathematics and Statistics \\ Lancaster University \\ Lancaster LA1 4YF \\ England \\ E-mail: r.m.green@lancaster.ac.uk \\ Department of Mathematics \\ Long Island University \\ Brookville, NY 11548 \\ USA \\ E-mail: losonczy@e-math.ams.org
}

\begin{abstract}
We investigate the compatibility of the set of fully commutative elements of a Coxeter group with the various types of Kazhdan-Lusztig cells using a canonical basis for a generalized version of the Temperley-Lieb algebra.

Cellules pleinement commutatives de Kazhdan-Lusztig

Nous étudions la compatibilité entre l'ensemble des éléments pleinement commutatifs d'un groupe de Coxeter et les divers types de cellules de Kazhdan-Lusztig, en utilisant une base canonique pour une version généralisée de l'algèbre de TemperleyLieb.
\end{abstract}

Key Words: canonical basis, cell theory, Coxeter group, Hecke algebra, KazhdanLusztig basis, Temperley-Lieb algebra

\section{To appear in Annales de l'Institut Fourier}

The first author was supported in part by a NUF-NAL award from the Nuffield Foundation.

Typeset by $\mathcal{A M}_{\mathcal{M}}$ - $\mathrm{TEX}_{\mathrm{E}}$ 


\section{INTRODUCTION}

The fully commutative elements, $W_{c}$, of a Coxeter group $W$ may be defined, following $[\mathbf{1 7}]$, as the set of elements $w$ with the property that any reduced expression for $w$ may be obtained from any other by a sequence of interchanges of adjacent, commuting Coxeter generators. These elements arise naturally in connection with the generalized Temperley-Lieb algebras defined in the simply laced case by Fan [2] and in general by Graham [8].

Kazhdan and Lusztig [14] have defined partitions of a Coxeter group $W$ arising from each of three equivalence relations, $\sim_{L}, \sim_{R}$ and $\sim_{L R}$. The corresponding equivalence classes of $W$ are known as left cells, right cells and two-sided cells, and it follows easily from the definitions that two-sided cells are unions of left (respectively, right) cells.

In this paper, we are concerned with the compatibility of the set $W_{c}$ with the various Kazhdan-Lusztig cells. More precisely, we wish to know when $W_{c}$ is a union of (left, right, or two-sided) cells. Our most general result is Theorem 2.2.3, where compatibility of $W_{c}$ with the (left, right, or two-sided) Kazhdan-Lusztig cells is shown to be related to several other conditions having to do with the TemperleyLieb quotient of the associated Hecke algebra.

It has been known at least since the theses of Fan and Graham ([2, Proposition 6], [8]) that $W_{c}$ is a union of two-sided cells in type $A$. In Graham's thesis a counterexample is given which shows that this is not the case in type $E$. (See also Example 2.2.5.) Our main task in $\S 3$ is to prove that there is full compatibility between $W_{c}$ and the Kazhdan-Lusztig cells in type $B$. We have also verified this property for Coxeter groups of types $F_{4}, H_{3}$, and $H_{4}$.

These results are reminiscent of some work of Fan and Stembridge [4, §3], who showed that in types $A, D, E$ and affine $A$, the set $W_{c}$ is a union of SpaltensteinSpringer-Steinberg cells.

We point out that our methods of proof are combinatorial and based on our previous work on IC-type ("canonical") bases for generalized Temperley-Lieb algebras 
$[\mathbf{1 1}, \mathbf{1 2}]$. In types $A, D$ and $E$, the canonical basis defined in $[\mathbf{1 1}]$ is a cellular basis, as defined by Graham in $[\mathbf{8}, \S 4]$.

As a consequence of the results in this paper, it becomes possible to describe the fully commutative cells in type $B$ very explicitly by using the diagram calculus for canonical basis elements in type $B$, which was given by the first author in $[\mathbf{1 0}$, Theorem 2.2.5].

\section{KAZHDAN-Lusztig BASES AND CELlS}

\subsection{Kazhdan-Lusztig bases.}

We begin by recalling the well-known basic properties of Hecke algebras arising from Coxeter systems. These properties all follow easily from the results of [14].

Let $X$ be a Coxeter graph, of arbitrary type, and let $W=W(X)$ be the associated Coxeter group with distinguished set of generating involutions $S=S(X)$. Denote by $<$ the Bruhat-Chevalley ordering on $W$. Let $\mathcal{A}=\mathbb{Z}\left[v, v^{-1}\right]$, let $\mathcal{A}^{-}=$ $\mathbb{Z}\left[v^{-1}\right]$ and let $q=v^{2}$.

We denote by $\mathcal{H}=\mathcal{H}(X)$ the Hecke algebra associated with $W$. As an $\mathcal{A}$-module, the Hecke algebra has a basis consisting of elements $T_{w}$, with $w$ ranging over $W$, that satisfy

$$
T_{s} T_{w}= \begin{cases}T_{s w} & \text { if } \ell(s w)>\ell(w), \\ q T_{s w}+(q-1) T_{w} & \text { if } \ell(s w)<\ell(w),\end{cases}
$$

where $\ell$ is the length function on the Coxeter group $W, w \in W$, and $s \in S$. It will be convenient to work with a second $\mathcal{A}$-basis $\left\{\widetilde{T}_{w}: w \in W\right\}$ for $\mathcal{H}$, which we obtain by defining $\widetilde{T}_{w}=v^{-\ell(w)} T_{w}$.

Definition 1.1.1. We denote by $a \mapsto \bar{a}$ the $\mathbb{Z}$-linear involution on the ring $\mathcal{A}$ that exchanges $v$ and $v^{-1}$. This can be extended to a $\mathbb{Z}$-linear involution $h \mapsto \bar{h}$ on $\mathcal{H}$, by defining $\overline{\sum_{w \in W} a_{w} T_{w}}=\sum_{w \in W} \overline{a_{w}} T_{w^{-1}}^{-1}$.

The Kazhdan-Lusztig basis may be characterized as follows.

Theorem 1.1.2 (Kazhdan-Lusztig). There is a unique $\mathcal{A}$-basis $\left\{C_{w}^{\prime}: w \in W\right\}$ for $\mathcal{H}$ such that 
(i) $\overline{C_{w}^{\prime}}=C_{w}^{\prime}$ for all $w \in W$;

(ii) $C_{w}^{\prime}=\sum_{x \in W} \widetilde{P}_{x, w} \widetilde{T}_{x}$, where each $\widetilde{P}_{x, w}$ lies in $\mathcal{A}^{-}$and satisfies

$$
\widetilde{P}_{x, w}= \begin{cases}1 & \text { if } x=w, \\ 0 & \text { if } x \not \leq w, \\ 0 \bmod v^{-1} \mathcal{A}^{-} & \text {if } x<w .\end{cases}
$$

Proof. This is a restatement of $[\mathbf{1 4},(1.1 \mathrm{c})]$. The relationship between $\widetilde{P}_{x, w}$ and the Kazhdan-Lusztig polynomials $P_{x, w}$ of $[\mathbf{1 4}]$ is $\widetilde{P}_{x, w}=v^{\ell(x)-\ell(w)} P_{x, w}$.

Theorem 1.1.2 leads to the following reformulation of [14, Definition 1.2].

Definition 1.1.3. Let $x, w \in W$ satisfy $x<w$. Define $\mu(x, w) \in \mathbb{Z}$ to be coefficient of $v^{-1}$ in $\widetilde{P}_{x, w}$ (i.e., the coefficient of $v^{\ell(w)-\ell(x)-1}$ in the Kazhdan-Lusztig polynomial $\left.P_{x, w}\right)$. If $x<w$ and $\mu(x, w) \neq 0$, then we write $x \prec w$.

\subsection{Kazhdan-Lusztig cells.}

The structure constants of $\mathcal{H}$ with respect to the Kazhdan-Lusztig basis of $\S 1.1$ give rise to various natural partitions of the group $W$ into "cells".

Although these structure constants are subtle, the product of two KazhdanLusztig basis elements may be computed in important special cases by appealing to the following well-known formula, which is implicit in $[\mathbf{1 4}, \S 2.2]$.

Proposition 1.2.1 (Kazhdan-Lusztig). Let $s, w \in W$ with $s \in S$. Then

$$
C_{s}^{\prime} C_{w}^{\prime}= \begin{cases}C_{s w}^{\prime}+\sum_{\substack{x \prec w \\ s x<x}} \mu(x, w) C_{x}^{\prime} & \text { if } s w>w, \\ \left(v+v^{-1}\right) C_{w}^{\prime} & \text { otherwise }\end{cases}
$$

where $\mu(x, w)$ is as in Definition 1.1.3.

This formula motivates the following definitions.

Definition 1.2.2. Let $x, w \in W$. We write $x \leq_{L} w$ if there is a chain

$$
x=x_{0}, x_{1}, \ldots, x_{r}=w
$$


possibly with $r=0$, such that for each $i<r, C_{x_{i}}^{\prime}$ occurs with nonzero coefficient in the linear expansion of $C_{s}^{\prime} C_{x_{i+1}}^{\prime}$ for some $s \in S$ such that $s x_{i+1}>x_{i+1}$. (By Proposition 1.2.1, this implies $s x_{i}<x_{i}$.)

This transitive preorder yields an equivalence relation $\sim_{L}$ on $W$ (where $x \sim_{L} w$ if and only if $x \leq_{L} w$ and $\left.w \leq_{L} x\right)$ whose equivalence classes are called the left cells of $W$. The preorder $\leq_{R}$ on $W$ is defined by the condition $x \leq_{R} w \Leftrightarrow x^{-1} \leq_{L} w^{-1}$,

and the preorder $\leq_{L R}$ is that generated by $\leq_{L}$ and $\leq_{R}$. These preorders yield equivalence relations $\sim_{R}$ and $\sim_{L R}$ on $W$ whose equivalence classes are called right cells and two-sided cells, respectively.

Remark 1.2.3. It is well known that the definition of $\leq_{L}$ given above agrees with the original definition in [14]. This follows from Proposition 1.2.1 and part (a) of the proof of [13, Proposition 7.15].

Remark 1.2.4. It is immediate from the construction of the left (respectively right, two-sided) cells that they are partially ordered via $\leq_{L}$ (respectively $\left.\leq_{R}, \leq_{L R}\right)$.

\section{Generalized Temperley-Lieb Algebras}

\subsection{Canonical bases for generalized Temperley-Lieb algebras.}

Let $X$ be a Coxeter graph, of arbitrary type. Let $\mathcal{J}=\mathcal{J}(X)$ be the two-sided ideal of $\mathcal{H}$ generated by the elements

$$
\sum_{w \in\left\langle s, s^{\prime}\right\rangle} T_{w}
$$

where $\left(s, s^{\prime}\right)$ runs over all pairs of elements of $S$ that correspond to adjacent nodes in the Coxeter graph. (If the nodes corresponding to $\left(s, s^{\prime}\right)$ are connected by a bond of infinite strength, then we omit the corresponding relation.)

Definition 2.1.1. The generalized Temperley-Lieb algebra, $\mathcal{T L}=\mathcal{T L}(X)$, is the quotient $\mathcal{A}$-algebra $\mathcal{H} / \mathcal{J}$. We denote the corresponding epimorphism of algebras by $\theta: \mathcal{H} \longrightarrow \mathcal{T L}$. 
The algebra $\mathcal{T L}$ may be of finite or infinite rank, and may be of finite rank even when it is the quotient of a Hecke algebra of infinite rank. Graham [8, Theorem 7.1] classified the algebras of finite rank into seven infinite families: $A, B, D, E, F$, $H$ and $I$.

Definition 2.1.2. A product $w_{1} w_{2} \cdots w_{n}$ of elements $w_{i} \in W$ is called reduced if $\ell\left(w_{1} w_{2} \cdots w_{n}\right)=\sum_{i} \ell\left(w_{i}\right)$. We reserve the terminology reduced expression for reduced products $w_{1} w_{2} \cdots w_{n}$ in which every $w_{i} \in S$.

We call an element $w \in W$ fully commutative if it cannot be written as a reduced product $x_{1} w_{s s^{\prime}} x_{2}$, where $x_{1}, x_{2} \in W$ and $w_{s s^{\prime}}$ is the longest element of some parabolic subgroup $\left\langle s, s^{\prime}\right\rangle$ such that $s, s^{\prime} \in S$ do not commute. This definition is equivalent to the one given in the introduction (see [17, Proposition 1.1]).

We define the content of $w \in W$ to be the set $c(w)$ of Coxeter generators $s \in S$ that appear in some (any) reduced expression for $w$.

Denote by $W_{c}=W_{c}(X)$ the set of all elements of $W$ that are fully commutative.

Let $t_{w}$ denote the image of the basis element $T_{w} \in \mathcal{H}$ in the quotient $\mathcal{T} \mathcal{L}$.

Proposition 2.1.3. [8, Theorem 6.2] The set $\left\{t_{w}: w \in W_{c}\right\}$ is an $\mathcal{A}$-basis for the algebra $\mathcal{T} \mathcal{L}$.

We now recall a principal result of $[\mathbf{1 1}]$, which establishes a canonical basis for $\mathcal{T L}$. This basis is a direct analogue of the Kazhdan-Lusztig basis in $\S 1$, although the precise relationship between the two is not immediate.

Definition 2.1.4. The involution $h \mapsto \bar{h}$ on $\mathcal{H}$ induces a $\mathbb{Z}$-linear ring involution on $\mathcal{T} \mathcal{L}[\mathbf{1 1}$, Lemma 1.4]. We use the bar notation to represent this map, as well: $\overline{\sum_{w \in W_{c}} a_{w} t_{w}}=\sum_{w \in W_{c}} \overline{a_{w}} t_{w^{-1}}^{-1}$.

Let $\mathcal{L}$ be the free $\mathcal{A}^{-}$-submodule of $\mathcal{T} \mathcal{L}$ with basis $\left\{\widetilde{t}_{w}: w \in W_{c}\right\}$, where $\widetilde{t}_{w}=$ $v^{-\ell(w)} t_{w}$, and let $\pi: \mathcal{L} \longrightarrow \mathcal{L} / v^{-1} \mathcal{L}$ be the canonical projection. For each $w \in W$, we denote by $\mathcal{L}_{w}$ the free $\mathcal{A}^{-}$-submodule of $\mathcal{T L}$ with basis $\left\{\widetilde{t}_{x}: x \in W_{c}, x \leq w\right\}$.

Proposition 2.1.5. [11, Theorem 2.3] There exists a unique basis $\left\{c_{w}: w \in W_{c}\right\}$ for $\mathcal{L}$ such that $\overline{c_{w}}=c_{w}$ and $\pi\left(c_{w}\right)=\pi\left(\widetilde{t}_{w}\right)$ for all $w \in W_{c}$. 
We call $\left\{c_{w}: w \in W_{c}\right\}$ the canonical basis (or the $I C$ basis) of $\mathcal{T L}$. It depends on the $t$-basis, the involution on $\mathcal{T L}$ from above, and the $\mathcal{A}^{-}$-lattice $\mathcal{L}$.

Definition 2.1.6. If $s \in S$, we write $b_{s} \in \mathcal{T} \mathcal{L}$ for the element $v^{-1} t_{s}+v^{-1}$. The elements $b_{s}=\theta\left(C_{s}^{\prime}\right)$ generate $\mathcal{T} \mathcal{L}$ as an $\mathcal{A}$-algebra.

For each $w \in W_{c}$, it makes sense to define $b_{w}=b_{s_{1}} b_{s_{2}} \cdots b_{s_{n}}$, where $s_{1} s_{2} \cdots s_{n}$ is any reduced expression for $w$. It is known that the set $\left\{b_{w}: w \in W_{c}\right\}$ is an $\mathcal{A}$-basis for $\mathcal{T} \mathcal{L}$; we call it the monomial basis. (In types $A, D$ and $E$, this agrees with Graham's cellular basis for $\mathcal{T} \mathcal{L}$ as defined in [8].)

For each $w \in W$, we denote by $\mathcal{L}_{w}^{\prime}$ the free $\mathcal{A}^{-}$-submodule of $\mathcal{T} \mathcal{L}$ with basis $\left\{b_{x}: x \in W_{c}, x \leq w\right\}$. We shall study the $\mathcal{A}^{-}$-lattices $\mathcal{L}_{w}^{\prime}$ in $\S 3.3$.

\subsection{Some general results.}

One of the main obstructions to understanding the relationship between the Kazhdan-Lusztig basis of $\mathcal{H}(X)$ and the canonical basis of $\mathcal{T L}(X)$ is that the set $W_{c}(X)$ may not be compatible with the two-sided cells. When a particular type of compatibility is present, the relationship between the two bases becomes transparent [12, Proposition 1.2.3]. It will be shown in $\S 3$ that this is the case when $X$ is of type $B_{n}$.

Before restricting ourselves to type $B_{n}$, we shall say something more about the general problem. The following result is helpful in this context.

Lemma 2.2.1. The set $\left\{\theta\left(C_{w}^{\prime}\right): w \in W_{c}\right\}$ is an $\mathcal{A}$-basis for $\mathcal{T} \mathcal{L}$.

Proof. This follows easily from [11, Lemma 1.5].

Lemma 2.2.2. Let $c \in \mathcal{L}$ be such that $\pi(c)=0$ and $\bar{c}=c$. Then $c=0$.

Proof. Express $c=\sum_{w \in W_{c}} a_{w} c_{w}$ as an $\mathcal{A}^{-}$-linear combination of the canonical basis. Since $\pi(c)=0$, we must have $a_{w} \in v^{-1} \mathcal{A}^{-}$for all $w$. But

$$
c=\bar{c}=\sum_{w \in W_{c}} \overline{a_{w}} c_{w},
$$

which implies that all $a_{w}=0$. 
There is compatibility between the set $W_{c}$ and the Kazhdan-Lusztig cells when the equivalent conditions of the following theorem are satisfied.

Theorem 2.2.3. Let $X$ be an arbitrary Coxeter graph, and maintain the usual notation, e.g., $\mathcal{J}=\mathcal{J}(X), W=W(X)$, etc. Then the following are equivalent:

(i) The ideal $\mathcal{J}$ is spanned by those elements $C_{w}^{\prime}$ that it contains.

(ii) The ideal $\mathcal{J}$ is spanned by the set $\left\{C_{w}^{\prime}: w \in W \backslash W_{c}\right\}$.

(iii) For each $w \in W \backslash W_{c}$, one has $\theta\left(C_{w}^{\prime}\right)=0$.

(iv) If $w \in W$, then $\theta\left(C_{w}^{\prime}\right) \in \mathcal{L}$ and

$$
\pi\left(\theta\left(C_{w}^{\prime}\right)\right)= \begin{cases}\pi\left(c_{w}\right) & \text { if } w \in W_{c} \\ 0 & \text { otherwise }\end{cases}
$$

(v) For each $w \in W \backslash W_{c}$, one has $\theta\left(\widetilde{T}_{w}\right) \in v^{-1} \mathcal{L}$.

(vi) The set $W \backslash W_{c}$ is closed under $\leq_{L}$ and so is a union of left cells.

(vii) The set $W \backslash W_{c}$ is closed under $\leq_{L R}$ and so is a union of two-sided cells.

(viii) The set $W_{c}$ is closed under $\geq_{L R}$ and so is a union of two-sided cells.

Note. If $\leq_{\Lambda}$ is a preorder on a set $\Lambda$ and $\Lambda^{\prime} \subseteq \Lambda$, then the statement " $\Lambda^{\prime}$ is closed under $\leq_{\Lambda}$ " means that whenever $\lambda_{1} \in \Lambda^{\prime}$ and $\lambda_{2} \in \Lambda$ are such that $\lambda_{2} \leq_{\Lambda} \lambda_{1}$, we have $\lambda_{2} \in \Lambda^{\prime}$.

Proof. We begin with the equivalence of (i), (ii) and (iii). The statement (i) implies that the set $\left\{\theta\left(C_{w}^{\prime}\right): \theta\left(C_{w}^{\prime}\right) \neq 0\right\}$ forms an $\mathcal{A}$-basis for $\mathcal{T} \mathcal{L}$. It follows that this set must equal the set in Lemma 2.2.1, which implies (ii). It is clear that (ii) implies (iii). If (iii) holds then $\mathcal{J}$ must contain all elements $C_{w}^{\prime}$ with $w \in W \backslash W_{c}$. However, $\mathcal{J}$ cannot be any bigger than the span of these elements by Lemma 2.2.1, so (i) follows.

(iii) $\Leftrightarrow$ (iv). Assume (iii) holds. It is clear that if $w \notin W_{c}$, then $\theta\left(C_{w}^{\prime}\right) \in \mathcal{L}$ and $\pi\left(\theta\left(C_{w}^{\prime}\right)\right)=0$. The remaining case is dealt with by the proof of $[\mathbf{1 2}$, Proposition 1.2.3]. Now assume (iv) and let $w \notin W_{c}$. Since the map $\theta$ is compatible (by [11, 
Lemma 1.4]) with the involutions on $\mathcal{H}$ and $\mathcal{T L}$ from above, we see that $\overline{\theta\left(C_{w}^{\prime}\right)}=$ $\theta\left(C_{w}^{\prime}\right)$. Since $\pi\left(\theta\left(C_{w}^{\prime}\right)\right)=0$, Lemma 2.2 .2 gives (iii).

(iv) $\Leftrightarrow(\mathrm{v})$. Note that if $w \in W_{c}$, then $\pi\left(\theta\left(\widetilde{T}_{w}\right)\right)=\pi\left(\widetilde{t}_{w}\right)=\pi\left(c_{w}\right)$ by the definition of the canonical basis. The equivalence of (iv) and (v) now follows from the fact that, relative to some total refinement of the Bruhat-Chevalley order, the (possibly infinite) change of basis matrices between the basis $\left\{C_{w}^{\prime}: w \in W\right\}$ and the basis $\left\{\widetilde{T}_{w}: w \in W\right\}$ are upper triangular with ones on the diagonal, and all the entries above the diagonal lie in $v^{-1} \mathcal{A}^{-}$.

(ii) $\Rightarrow$ (vi). By Definition 1.2.2, it is enough to check that if $w \notin W_{c}$ and $s \in S$ with $s w>w$, then all the terms occurring in the expansion of $C_{s}^{\prime} C_{w}^{\prime}$ in Proposition 1.2.1 are parametrized by elements $x \notin W_{c}$. By (ii), $w \notin W_{c}$ implies $C_{w}^{\prime} \in \mathcal{J}$. Since $\mathcal{J}$ is an ideal, $C_{s}^{\prime} C_{w}^{\prime} \in \mathcal{J}$. Another application of (ii) completes the proof.

(vi) $\Rightarrow$ (vii). Suppose $w \notin W_{c}$ and $x \leq_{R} w$, meaning that $x^{-1} \leq_{L} w^{-1}$. By symmetry of the definition of $W_{c}$, we have $w^{-1} \notin W_{c}$, and (vi) shows that $x^{-1} \notin W_{c}$, meaning that $x \notin W_{c}$. It follows that $W \backslash W_{c}$ is closed under $\leq_{R}$. Since $W \backslash W_{c}$ is closed under $\leq_{L}$ and $\leq_{R}$, it is closed under $\leq_{L R}$ and is therefore a union of two-sided cells.

(vii) $\Rightarrow$ (ii). Since $W \backslash W_{c}$ is closed under $\leq_{L R}$ and $\left\{C_{s}^{\prime}: s \in S\right\}$ is a set of algebra generators for $\mathcal{H}$, it follows that $\left\{C_{w}^{\prime}: w \notin W_{c}\right\}$ spans a two-sided ideal of $\mathcal{H}$. The generators of $\mathcal{J}$ (see $\S 2.1$ ) are of the form $v^{\ell(w)} C_{w}^{\prime}$ for certain $w \notin W_{c}$, so $\mathcal{J}$ is contained in this ideal. On the other hand, $\mathcal{J}$ contains $\left\{C_{w}^{\prime}: w \notin W_{c}\right\}$ by Lemma 2.2.1. Thus, condition (ii) holds.

The equivalence of (vii) and (viii) is obvious.

Example 2.2.4. Consider the finite dihedral case, or in other words, take $X=$ $I_{2}(m)$ for $m<\infty$. Let $w_{0}$ denote the longest element of $W\left(I_{2}(m)\right)$. The ideal $\mathcal{J}\left(I_{2}(m)\right)$ is spanned by the single Kazhdan-Lusztig basis element $C_{w_{0}}^{\prime}$. Thus, the equivalent conditions of Theorem 2.2.3 hold for finite dihedral groups. 
In the next section, it will be shown that the conditions of Theorem 2.2.3 hold when the underlying graph is of type $B_{n}$. Our proof will also handle type $A_{n}$ as a special case.

Example 2.2.5. Take the underlying graph $X$ to be of type $D_{n}(n \geq 4)$. Let $\sigma_{1}, \sigma_{2}, \ldots, \sigma_{n}$ denote the Coxeter generators, labelled so that $\sigma_{3}$ corresponds to the branch node and $\sigma_{1}, \sigma_{2}$ commute with all generators except $\sigma_{3}$. Consider the elements $w=\sigma_{2} \sigma_{3} \sigma_{4} \sigma_{3} \sigma_{1} \sigma_{2} \sigma_{3} \notin W_{c}\left(D_{n}\right)$ and $x=\sigma_{1} \sigma_{2} \sigma_{4} \sigma_{3} \in W_{c}\left(D_{n}\right)$. Observe that $\sigma_{1} w>w$. When $C_{\sigma_{1}}^{\prime} C_{w}^{\prime}$ is written as a linear combination of Kazhdan-Lusztig basis elements, the element $C_{x}^{\prime}$ appears with coefficient 1 . Thus, $x \leq_{L} w$, so that condition (vi) fails when the underlying graph is of type $D_{n}$. Further computation reveals that $x \sim_{L} w$, which shows that $W_{c}\left(D_{n}\right)$ is not a union of left cells. This example also shows that condition (vi) is violated (and that $W_{c}$ is not a union of left cells) in types $E_{6}, E_{7}$ and $E_{8}$. The incompatibility of $W_{c}$ with Kazhdan-Lusztig cells in type $E$ was described explicitly in [8, $\S 9.9]$.

We remark that it is nevertheless true that the image under $\theta$ of the set of all $C_{u}^{\prime}$ indexed by $u \in W_{c}\left(D_{n}\right)$ equals the canonical basis of $\mathcal{T} \mathcal{L}\left(D_{n}\right)$ (see $[\mathbf{1 5}$, Theorem $3.4]$ ). It is not known whether the corresponding statement holds in type $E$.

\section{TYPE B}

In this section, we study the compatibility of cells and fully commutative elements when the underlying Coxeter graph $X$ is of type $B_{n}$.

\subsection{Statement of results.}

Our main objective in $\S 3$ is to prove the following

Theorem 3.1.1. When the underlying Coxeter graph is of type $B_{n}$, the equivalent conditions of Theorem 2.2.3 are satisfied. In particular, the set $W_{c}\left(B_{n}\right)$ is closed under $\geq_{L R}$ and so is a union of two-sided Kazhdan-Lusztig cells.

This result is a strengthening of [12, Theorem 2.2.1], where it was shown that $\theta\left(C_{w}^{\prime}\right) \in \mathcal{L}$ for all $w \in W\left(B_{n}\right)$, and thus that the basis in Lemma 2.2.1 for $\mathcal{T} \mathcal{L}\left(B_{n}\right)$ 
agrees with the canonical basis of $\mathcal{T} \mathcal{L}\left(B_{n}\right)$.

Theorem 3.1.1 also implies the corresponding statement for type $A_{n}$, which was previously known (see [3, Proposition 3.1.1]).

Corollary 3.1.2. When the underlying Coxeter graph is of type $A_{n}$, the equivalent conditions of Theorem 2.2.3 are satisfied.

Proof. Identify the Coxeter group $W\left(A_{n}\right)$ with the parabolic subgroup of the Coxeter group $W\left(B_{n+1}\right)$ that corresponds to omission of the appropriate end generator.

Let $s \in S\left(A_{n}\right)$ and $w \in W\left(A_{n}\right)$ be such that $w \notin W_{c}\left(A_{n}\right)$ and $s w>w$. By considering $C_{s}^{\prime} C_{w}^{\prime}$ and using condition (vi) of Theorem 2.2.3 applied to type $B_{n+1}$, we see that condition (vi) holds for type $A_{n}$.

The remaining cases arising from finite irreducible Coxeter groups are $F_{4}, H_{3}$, and $H_{4}$. A series of computer calculations using du Cloux's program "Coxeter" [1] shows that condition (vi) of Theorem 2.2.3 holds in each of these cases.

We can summarize our results as follows.

Corollary 3.1.3. Let $X$ be a Coxeter graph such that $W(X)$ is finite and irreducible. Then $W_{c}(X)$ is a union of two-sided Kazhdan-Lusztig cells if and only if $X$ does not contain $D_{4}$ as a subgraph.

\subsection{Some combinatorial preparation.}

The following proposition describes a useful way to parse certain reduced expressions. A proof can be found in [12, Lemma 2.1.2].

Proposition 3.2.1. Let $w \in W_{c}\left(B_{n}\right)$ and $s \in S\left(B_{n}\right)$ satisfy $w s \notin W_{c}\left(B_{n}\right)$. There exists a unique $s^{\prime} \in S\left(B_{n}\right)$ such that any reduced expression for $w$ can be parsed in one of the following two ways.

(i) $w=w_{1} s w_{2} s^{\prime} w_{3}$, where $s s^{\prime}$ has order 3 , and $s$ commutes with every member of $c\left(w_{2}\right) \cup c\left(w_{3}\right)$;

(ii) $w=w_{1} s^{\prime} w_{2} s w_{3} s^{\prime} w_{4}$, where $s s^{\prime}$ has order $4, s$ commutes with every member of $c\left(w_{3}\right) \cup c\left(w_{4}\right)$, and $s^{\prime}$ commutes with every member of $c\left(w_{2}\right) \cup c\left(w_{3}\right)$. 
The algebra $\mathcal{T} \mathcal{L}\left(B_{n}\right)$ is known to be generated by the monomial elements $b_{s}$, with $s$ ranging over all Coxeter generators, subject to the following relations: $b_{s}^{2}=q_{c} b_{s}$, where $q_{c}=[2]=v+v^{-1} ; b_{s} b_{s^{\prime}}=b_{s^{\prime}} b_{s}$ if $s, s^{\prime}$ commute; $b_{s} b_{s^{\prime}} b_{s}=b_{s}$ if $s s^{\prime}$ has order $3 ; b_{s} b_{s^{\prime}} b_{s} b_{s^{\prime}}=2 b_{s} b_{s^{\prime}}$ if $s s^{\prime}$ has order $4($ see $[\mathbf{9}, \S 1])$.

Lemma 3.2.2. Let $w \in W\left(B_{n}\right)$ and let $s_{1} s_{2} \cdots s_{m}$ be a reduced expression for $w$. Given integers $1 \leq i_{1}<i_{2}<\cdots<i_{k} \leq m$, we have $b_{s_{i_{1}}} b_{s_{i_{2}}} \cdots b_{s_{i_{k}}}=a q_{c}^{\mu} b_{w^{\prime}}$, where $a$ and $\mu$ are nonnegative integers and $w^{\prime} \in W_{c}\left(B_{n}\right)$. Moreover, we have $w^{\prime} \leq w$ and $\ell\left(w^{\prime}\right) \leq k$.

Proof. This follows by a simple induction on $k$, using the subexpression characterization of Bruhat-Chevalley order together with Proposition 3.2.1 and the relations for the monomial generators given in the previous paragraph.

Remark 3.2.3. We usually apply Lemma 3.2.2 in the following way. Let $w=x y z$ be a reduced product, and consider $b_{x} \widetilde{t}_{y} b_{z}$. We would like to know that this is a linear combination of monomial basis elements $b_{u}$ with $u \leq w$. To see that this is the case, let $s_{1} s_{2} \cdots s_{m}$ be a reduced expression for $y$. Then $b_{x} \widetilde{t}_{y} b_{z}$ equals

$$
b_{x} \widetilde{t}_{s_{1}} \widetilde{t}_{s_{2}} \cdots \widetilde{t}_{s_{m}} b_{z}=b_{x}\left(b_{s_{1}}-v^{-1}\right)\left(b_{s_{2}}-v^{-1}\right) \cdots\left(b_{s_{m}}-v^{-1}\right) b_{z}
$$

and we see that this expands into a combination of $b_{u}$ with $u \leq w$ by Lemma 3.2.2.

The next result gives useful information concerning the structure constants for the monomial basis; it will be used repeatedly in $\S 3.3$.

Proposition 3.2.4. [12, Lemma 2.1.3] Let $w \in W_{c}\left(B_{n}\right)$ and let $s \in S\left(B_{n}\right)$. We have $b_{w} b_{s}=a q_{c}^{\mu} b_{w^{\prime}}$ for some fully commutative $w^{\prime}$ and some nonnegative integers $a$ and $\mu$. Furthermore, one has (i) $\mu \leq 1$; (ii) $\ell\left(w^{\prime} s\right)<\ell\left(w^{\prime}\right)$; (iii) $\mu=0$ if $\ell\left(w s^{\prime}\right)<\ell(w)$ for some $s^{\prime} \in S\left(B_{n}\right)$ that does not commute with $s$.

Let $\sigma_{1}, \sigma_{2}, \ldots, \sigma_{n}$ be the elements of $S\left(B_{n}\right)$, labelled so that $\sigma_{1} \sigma_{2}$ has order 4 and $\sigma_{i} \sigma_{i+1}$ has order 3 for all $i>1$. Define, for each $1 \leq r \leq n$, the set $W^{(r)}=\left\{w \in W\left(B_{r}\right): i<r \Rightarrow \ell\left(\sigma_{i} w\right)>\ell(w)\right\}$. It is known that $W^{(r)}$ is a 
system of right coset representatives for the parabolic subgroup $W\left(B_{r-1}\right)$ of $W\left(B_{r}\right)$. Moreover, one has $\ell(x y)=\ell(x)+\ell(y)$ for all $x \in W\left(B_{r-1}\right)$ and $y \in W^{(r)}$ (see $[\mathbf{1 3}$, $\S 5.12])$. Thus, each $y \in W^{(r)}$ is the unique element of minimum length in the coset $W\left(B_{r-1}\right) y$. The elements of $W^{(r)}$ are given as follows:

$$
\begin{gathered}
\left\{e, \sigma_{r}, \sigma_{r} \sigma_{r-1}, \ldots, \sigma_{r} \sigma_{r-1} \cdots \sigma_{2} \sigma_{1}, \sigma_{r} \sigma_{r-1} \cdots \sigma_{2} \sigma_{1} \sigma_{2}, \ldots,\right. \\
\left.\sigma_{r} \sigma_{r-1} \cdots \sigma_{2} \sigma_{1} \sigma_{2} \cdots \sigma_{r-1} \sigma_{r}\right\} .
\end{gathered}
$$

Note that each element of $W^{(r)}$ has a unique reduced expression and hence is fully commutative.

Any $w \in W\left(B_{n}\right)$ can be written uniquely as a product $w_{1} w_{2} \cdots w_{n}$, where each $w_{i} \in W^{(i)}$. By the previous paragraph, this product is reduced. Thus, if we delete each $w_{i}$ that equals the identity, and then replace each of the remaining $w_{i}$ with its unique reduced expression, we obtain a "normal" reduced expression for $w$.

We frequently use without comment the following consequence of the Exchange Condition, which is valid for any Coxeter system: if $w \in W$ and $s \in S$, then $w$ has a reduced expression ending in $s$ if and only if $\ell(w s)<\ell(w)($ see $[\mathbf{1 3}, \S 5.8])$.

\subsection{The $\mathcal{A}^{-}$-lattices $\mathcal{L}_{w}^{\prime}$.}

In the following series of lemmas, we study the $\mathcal{A}^{-}$-lattices in $\mathcal{T L}\left(B_{n}\right)$ of the form $\mathcal{L}_{w}^{\prime}$ (recall Definition 2.1.6). Our goal, which is accomplished in Proposition 3.3.10, is to prove that $\widetilde{t}_{w} \in v^{-1} \mathcal{L}_{w}^{\prime}$ whenever $w \notin W_{c}\left(B_{n}\right)$. This will enable us to establish condition (v) of Theorem 2.2.3 for the case where $X=B_{n}$.

Lemma 3.3.1. Let $x \in W_{c}\left(B_{n-1}\right)$, let $w \in W^{(n)}$ and let $k \geq 0$. Suppose that there exist $s, s^{\prime} \in S\left(B_{n}\right)$, with $\ell\left(w s s^{\prime}\right)<\ell(w s)<\ell(w)$, such that $b_{x} \widetilde{t}_{w s s^{\prime}} \in v^{-k} \mathcal{L}_{x w s s^{\prime}}^{\prime}$ and $b_{x} \widetilde{t}_{w s} \in v^{-k} \mathcal{L}_{x w s}^{\prime}$. Then $b_{x} \widetilde{t}_{w} \in v^{-k} \mathcal{L}_{x w}^{\prime}$.

Proof. First note that when $b_{x} \widetilde{t}_{w}$ is written as a linear combination of monomial basis elements $b_{y}$, the coefficient of $b_{y}$ is nonzero only if $y \leq x w$ by Remark 3.2.3. Thus, we may turn our attention to the degrees of the various coefficients. 
By hypothesis, we can write $b_{x} \widetilde{t}_{w s s^{\prime}}$ as a sum of terms $a_{y} b_{y}$ with each $a_{y} \in$ $v^{-k} \mathcal{A}^{-}$. Since

$$
b_{x} \widetilde{t}_{w s}=b_{x} \widetilde{t}_{w s s^{\prime}} \widetilde{t}_{s^{\prime}}=b_{x} \widetilde{t}_{w s s^{\prime}}\left(b_{s^{\prime}}-v^{-1}\right)
$$

we see that $b_{x} \widetilde{t}_{w s}$ equals a sum of terms of the form $a_{y} b_{y}\left(b_{s^{\prime}}-v^{-1}\right)$, where $a_{y} \in$ $v^{-k} \mathcal{A}^{-}$. We can use this fact together with Proposition 3.2.4 (ii) and the hypothesis on $b_{x} \widetilde{t}_{w s}$ to deduce that when $b_{x} \widetilde{t}_{w s}$ is written as a linear combination of monomial basis elements $b_{z}$, either the coefficient of $b_{z}$ lies in $v^{-k-1} \mathcal{A}^{-}$, or the coefficient lies in $v^{-k} \mathcal{A}^{-}$and $\ell\left(z s^{\prime}\right)<\ell(z)$.

Now consider the equalities

$$
b_{x} \tilde{t}_{w}=b_{x} \widetilde{t}_{w s} \widetilde{t}_{s}=b_{x} \widetilde{t}_{w s}\left(b_{s}-v^{-1}\right) .
$$

In view of the previous paragraph, together with the fact that $s, s^{\prime}$ do not commute (since $w$ has a unique reduced expression), parts (i) and (iii) of Proposition 3.2.4 enable us to conclude that $b_{x} \widetilde{t}_{w}$ is a linear combination of monomial basis elements $b_{z^{\prime}}\left(z^{\prime} \leq x w\right)$ with coefficients in $v^{-k} \mathcal{A}^{-}$, as desired.

Lemma 3.3.2. Let $x \in W_{c}\left(B_{n-1}\right)$ and let $w \in W^{(n)}$. Then $b_{x} \widetilde{t}_{w} \in \mathcal{L}_{x w}^{\prime}$.

Proof. We argue by induction on $\ell(w)$. The lemma is obviously true for $\ell(w)=0$, and if $\ell(w)=1$, then $w=\sigma_{n} \notin c(x)$. Hence, $b_{x} \widetilde{t}_{w}=b_{x}\left(b_{\sigma_{n}}-v^{-1}\right)=b_{x \sigma_{n}}-v^{-1} b_{x}$, and one sees that this last expression belongs to $\mathcal{L}_{x w}^{\prime}$.

Suppose that $\ell(w)>1$. There exist (uniquely determined) Coxeter generators $s, s^{\prime}$ such that $\ell\left(w s s^{\prime}\right)<\ell(w s)<\ell(w)$. By the inductive hypothesis, $b_{x} \widetilde{t}_{w s s^{\prime}} \in$ $\mathcal{L}_{x w s s^{\prime}}^{\prime}$ and $b_{x} \widetilde{t}_{w s} \in \mathcal{L}_{x w s}^{\prime}$. But then $b_{x} \widetilde{t}_{w} \in \mathcal{L}_{x w}^{\prime}$ by Lemma 3.3.1 (taking $k=0$ ). The inductive step is complete.

Proposition 3.3.3. We have $\widetilde{t}_{w} \in \mathcal{L}_{w}^{\prime}$ for all $w \in W\left(B_{n}\right)$.

Proof. We proceed by induction on $\ell(w)$. If $\ell(w)=0$, then $w=e$ and we have $\widetilde{t}_{e}=b_{e}$. Suppose that $\ell(w)>0$. Let $r>0$ be the smallest integer such that $w \in W\left(B_{r}\right)$. Write $w$ as a reduced product $w=y z$, where $y \in W\left(B_{r-1}\right)$ and 
$z \in W^{(r)}$. We have $\widetilde{t}_{w}=\widetilde{t}_{y} \widetilde{t}_{z}$. By the inductive hypothesis, we may write $\widetilde{t}_{y}$ as a linear combination of monomial basis elements $b_{x}(x \leq y)$ with coefficients in $\mathcal{A}^{-}$. Thus, $\widetilde{t}_{w}$ equals a linear combination of products of the form $b_{x} \widetilde{t}_{z}(x \leq y)$, with coefficients in $\mathcal{A}^{-}$.

If we can show that any such product $b_{x} \widetilde{t}_{z}$ lies in $\mathcal{L}_{w}^{\prime}$, then the inductive step will be established. But Lemma 3.3.2 gives us $b_{x} \widetilde{t}_{z} \in \mathcal{L}_{x z}^{\prime}$, and the subexpression characterization of Bruhat-Chevalley order gives $x z \leq w$. The proof is complete.

The following two lemmas are needed to handle certain cases that arise in the proofs of lemmas 3.3.6 and 3.3.8.

Lemma 3.3.4. Let $x \in W_{c}\left(B_{n-1}\right)$ and let $w, w^{\prime} \in W^{(n)}$. Suppose that $w=w^{\prime} u$ (reduced) for some $u \in W\left(B_{n}\right)$. Let $u^{\prime} \in W\left(B_{n}\right)$ satisfy $u^{\prime} \leq u$, and let $k \geq 1$. If $\ell\left(u^{\prime}\right)<k$, then $v^{-k} b_{x} \widetilde{t}_{w^{\prime}} b_{u^{\prime}} \in v^{-1} \mathcal{L}_{x w}^{\prime}$.

Proof. Fix a reduced expression $s_{1} s_{2} \cdots s_{m}$ for $u^{\prime}$. We assume that $m<k$. By Lemma 3.3.2, the product $b_{x} \widetilde{t}_{w^{\prime}}$ can be written as a sum of terms of the form $a_{y} b_{y}$, where $a_{y} \in \mathcal{A}^{-}$and $y \leq x w^{\prime}$. Thus, $v^{-k} b_{x} \widetilde{t}_{w^{\prime}} b_{u^{\prime}}$ equals a sum of terms of the form

$$
v^{-k} a_{y} b_{y} b_{u^{\prime}}=v^{-k} a_{y} b_{y} b_{s_{1}} b_{s_{2}} \cdots b_{s_{m}}
$$

where again $a_{y} \in \mathcal{A}^{-}$and $y \leq x w^{\prime}$.

By applying Proposition 3.2.4 (i) repeatedly ( $m$ times on the same term) and then applying Lemma 3.2.2, we find that $v^{-k} a_{y} b_{y} b_{s_{1}} b_{s_{2}} \cdots b_{s_{m}}$ lies in $v^{-1} \mathcal{L}_{x w}^{\prime}$.

Lemma 3.3.5. Let $x \in W_{c}\left(B_{n-1}\right)$ and let $w, w^{\prime} \in W^{(n)}$. Suppose that $w=w^{\prime} u$ (reduced) for some $u \in W\left(B_{n}\right)$. Let $u^{\prime} \in W\left(B_{n}\right)$ satisfy $u^{\prime} \leq u$, and assume that $u^{\prime}$ has a unique reduced expression $s_{1} s_{2} \cdots s_{m}$. Then $b_{x} \widetilde{t}_{w^{\prime}} b_{u^{\prime}} \in \mathcal{L}_{x w}^{\prime}$ if there exists an $s \in S\left(B_{n}\right)$ that does not commute with $s_{1}$ and that satisfies either (i) $\ell\left(w^{\prime} s\right)<\ell\left(w^{\prime}\right)$; or (ii) $\ell(x s)<\ell(x)$ and $s w^{\prime}=w^{\prime} s$.

Proof. We first point out that by Remark 3.2.3, when $b_{x} \widetilde{t}_{w^{\prime}} b_{u^{\prime}}$ is expressed as a linear combination of monomial basis elements, all nonzero terms correspond to $y \in W_{c}\left(B_{n}\right)$ that satisfy $y \leq x w$. 
Suppose that (i) holds. Write $w^{\prime}=w^{\prime \prime} s$ (reduced). We have $b_{x} \widetilde{t}_{w^{\prime}} \in \mathcal{L}_{x w^{\prime}}^{\prime}$ and $b_{x} \widetilde{t}_{w^{\prime \prime}} \in \mathcal{L}_{x w^{\prime \prime}}^{\prime}$ by Lemma 3.3.2. The equalities $b_{x} \widetilde{t}_{w^{\prime}}=b_{x} \widetilde{t}_{w^{\prime \prime}} \widetilde{t}_{s}=b_{x} \widetilde{t}_{w^{\prime \prime}}\left(b_{s}-v^{-1}\right)$ together with Proposition 3.2.4 (ii) imply that when $b_{x} \widetilde{t}_{w^{\prime}}$ is written as a sum of terms of the form $a_{y} b_{y}\left(a_{y} \in \mathcal{A}^{-}\right)$, for each $y$, either $\ell(y s)<\ell(y)$ or else $a_{y} \in v^{-1} \mathcal{A}^{-}$.

Thus, $b_{x} \widetilde{t}_{w^{\prime}} b_{u^{\prime}}$ is a sum of terms of the form $a_{y} b_{y} b_{u^{\prime}}=a_{y} b_{y} b_{s_{1}} b_{s_{2}} \cdots b_{s_{m}}$, with $y$ and $a_{y}$ as described above. If $\ell(y s)<\ell(y)$ then, since $s s_{1} \neq s_{1} s$ and $s_{i} s_{i+1} \neq$ $s_{i+1} s_{i}$ for all $i<m$, repeated applications of parts (ii) and (iii) of Proposition 3.2.4 give $a_{y} b_{y} b_{s_{1}} b_{s_{2}} \cdots b_{s_{m}} \in \mathcal{L}_{x w}^{\prime}$. On the other hand, if $a_{y} \in v^{-1} \mathcal{A}^{-}$, then $a_{y} b_{y} b_{s_{1}}=a^{\prime} b_{y^{\prime}}$ with $a^{\prime} \in \mathcal{A}^{-}$by Proposition 3.2 .4 (i). Also, $\ell\left(y^{\prime} s_{1}\right)<\ell\left(y^{\prime}\right)$ by part (ii) of the same proposition. Since $s_{i} s_{i+1} \neq s_{i+1} s_{i}$ for all $i<m$, we have $a_{y} b_{y} b_{s_{1}} b_{s_{2}} \cdots b_{s_{m}}=a^{\prime} b_{y^{\prime}} b_{s_{2}} \cdots b_{s_{m}} \in \mathcal{L}_{x w}^{\prime}$ (again by repeated applications of parts (ii) and (iii) of Proposition 3.2.4).

Suppose now that (ii) holds. Write $x=x^{\prime} s$ (reduced). Since $s w^{\prime}=w^{\prime} s$, we have $b_{x} \widetilde{t}_{w^{\prime}}=b_{x^{\prime}} \widetilde{t}_{w^{\prime}} b_{s}$; hence, by Proposition 3.2.4 (ii), when $b_{x} \widetilde{t}_{w^{\prime}}$ is written as a sum of terms of the form $a_{y} b_{y}$, we have $\ell(y s)<\ell(y)$ whenever $a_{y} \neq 0$. But then the reasoning from the previous paragraph gives $a_{y} b_{y} b_{s_{1}} b_{s_{2}} \cdots b_{s_{m}} \in \mathcal{L}_{x w}^{\prime}$.

The following lemma and Lemma 3.3.8 are needed to handle the inductive step of Lemma 3.3.9.

Lemma 3.3.6. Let $x \in W_{c}\left(B_{n-1}\right)$ and let $w \in W^{(n)}$. Suppose that there exists $s \in S\left(B_{n}\right)$, with $\ell(w s)<\ell(w)$, such that $x w s$ is fully commutative but $x w$ is not. Then $b_{x} \tilde{t}_{w} \in v^{-1} \mathcal{L}_{x w}^{\prime}$.

Proof. By the hypothesis of the lemma, together with Proposition 3.2.1, there are two possibilities concerning the nature of $w$ and $x$ : Either (a) $w=\sigma_{n} \sigma_{n-1} \cdots \sigma_{r+1} \sigma_{r}$ $(2 \leq r<n)$ and $\ell\left(x \sigma_{r}\right)<\ell(x)$; or (b) $w=\sigma_{n} \sigma_{n-1} \cdots \sigma_{2} \sigma_{1} \sigma_{2}$ and $\ell\left(x \sigma_{1}\right)<\ell(x)$.

Suppose that case (a) holds. Let $w^{\prime}=w \sigma_{r} \sigma_{r+1}$. We have

$$
b_{x} \widetilde{t}_{w}=b_{x \sigma_{r}} b_{\sigma_{r}} \tilde{t}_{w^{\prime}} \widetilde{t}_{\sigma_{r+1}} \tilde{t}_{\sigma_{r}}=b_{x \sigma_{r}} \widetilde{t}_{w^{\prime}} b_{\sigma_{r}}\left(b_{\sigma_{r+1}}-v^{-1}\right)\left(b_{\sigma_{r}}-v^{-1}\right) .
$$

This last expression expands into the product

$$
b_{x \sigma_{r}} \widetilde{t}_{w^{\prime}}\left(b_{\sigma_{r}} b_{\sigma_{r+1}} b_{\sigma_{r}}-v^{-1} b_{\sigma_{r}} b_{\sigma_{r+1}}-v^{-1} b_{\sigma_{r}}^{2}+v^{-2} b_{\sigma_{r}}\right) .
$$


Using the relations for the monomial generators given in $§ 3.2$, we may simplify this last expression to

$$
b_{x \sigma_{r}} \widetilde{t}_{w^{\prime}}\left(-v^{-1} b_{\sigma_{r}} b_{\sigma_{r+1}}\right)=b_{x} \widetilde{t}_{w^{\prime}}\left(-v^{-1} b_{\sigma_{r+1}}\right) .
$$

Thus, $b_{x} \widetilde{t}_{w}=-v^{-1} b_{x} \widetilde{t}_{w^{\prime}} b_{\sigma_{r+1}}$. This last expression is easily seen to lie in $v^{-1} \mathcal{L}_{x w}^{\prime}$ by Lemma 3.3.5 (ii) (taking $s=\sigma_{r}$ ).

We turn to case (b), wherein $w=\sigma_{n} \sigma_{n-1} \cdots \sigma_{2} \sigma_{1} \sigma_{2}$ and $x$ has a reduced expression ending in $\sigma_{1}$. Let $w^{\prime \prime}=w \sigma_{2} \sigma_{1} \sigma_{2}$. Observe that $b_{x} \widetilde{t}_{w}$ equals

$$
b_{x \sigma_{1}} b_{\sigma_{1}} \widetilde{t}_{w^{\prime \prime}} \widetilde{t}_{\sigma_{2}} \widetilde{t}_{\sigma_{1}} \widetilde{t}_{\sigma_{2}}=b_{x \sigma_{1}} \widetilde{t}_{w^{\prime \prime}} b_{\sigma_{1}}\left(b_{\sigma_{2}}-v^{-1}\right)\left(b_{\sigma_{1}}-v^{-1}\right)\left(b_{\sigma_{2}}-v^{-1}\right) .
$$

By expanding and then simplifying this last expression, we obtain

$$
b_{x \sigma_{1}} \widetilde{t}_{w^{\prime \prime}}\left(-v^{-1} b_{\sigma_{1}} b_{\sigma_{2}} b_{\sigma_{1}}+v^{-1} b_{\sigma_{1}}\right)=b_{x} \widetilde{t}_{w^{\prime \prime}}\left(-v^{-1} b_{\sigma_{2} \sigma_{1}}+v^{-1}\right) .
$$

Thus, $b_{x} \widetilde{t}_{w}=-v^{-1} b_{x} \widetilde{t}_{w^{\prime \prime}} b_{\sigma_{2} \sigma_{1}}+v^{-1} b_{x} \widetilde{t}_{w^{\prime \prime}}$. This last pair of terms lies in $v^{-1} \mathcal{L}_{x w}^{\prime}$ by Lemma 3.3 .5 (ii).

The next lemma is required to address a certain term that arises in the proof of Lemma 3.3.8.

Lemma 3.3.7. Let $x \in W_{c}\left(B_{n-1}\right)$ and let $w=\sigma_{n} \sigma_{n-1} \cdots \sigma_{r} \sigma_{r-1}$, with $2 \leq r<n$. Let $w^{\prime}=w \sigma_{r-1} \sigma_{r} \sigma_{r+1}$. Suppose that $\ell\left(x \sigma_{r}\right)<\ell(x)$. Then $b_{x} \widetilde{t}_{w^{\prime}} b_{\sigma_{r-1} \sigma_{r+1}} \in \mathcal{L}_{x w}^{\prime}$.

Proof. Note first that when $b_{x} \widetilde{t}_{w^{\prime}} b_{\sigma_{r-1} \sigma_{r+1}}$ is written as a linear combination of monomial basis elements $b_{y}$, every nonzero term is indexed by a $y$ satisfying $y \leq x w$ by Remark 3.2.3. If $w^{\prime}=e$, then $b_{x} \widetilde{t}_{w^{\prime}} b_{\sigma_{r-1} \sigma_{r+1}}=b_{x} b_{\sigma_{r-1}} b_{\sigma_{r+1}}$, which equals $a b_{y} b_{\sigma_{r+1}}$ for some integer $a$ and some $y \in W_{c}\left(B_{n-1}\right)$ by Proposition 3.2.4 (iii). Since $w^{\prime}=e$, we have $\sigma_{r+1}=\sigma_{n} \notin c(y)$, hence the product $y \sigma_{r+1}$ is reduced and fully commutative. Therefore, $a b_{y} b_{\sigma_{r+1}}=a b_{y \sigma_{r+1}} \in \mathcal{L}_{x w}^{\prime}$.

Now assume $w^{\prime} \neq e$. Let $w^{\prime \prime}=w^{\prime} \sigma_{r+2}$ and let $x^{\prime}=x \sigma_{r}$. By Lemma 3.3.2, we can write $b_{x} \widetilde{t}_{w^{\prime}}$ as a sum of terms of the form $a_{y} b_{y}$, where $y \leq x w^{\prime}$ and $a_{y} \in \mathcal{A}^{-}$. Moreover, we have $\ell\left(y \sigma_{r}\right)<\ell(y)$ whenever $a_{y} \neq 0$, owing to the equality $b_{x} \widetilde{t}_{w^{\prime}}=$ 
$b_{x^{\prime}} \widetilde{t}_{w^{\prime}} b_{\sigma_{r}}$ and Proposition 3.2.4 (ii). Thus, $b_{x} \widetilde{t}_{w^{\prime}} b_{\sigma_{r-1} \sigma_{r+1}}$ is a sum of terms of the form $a_{y} b_{y} b_{\sigma_{r-1} \sigma_{r+1}}$, where $y \leq x w^{\prime}$ and $\ell\left(y \sigma_{r}\right)<\ell(y)$ whenever $a_{y} \neq 0$.

We can say more about the terms $a_{y} b_{y} b_{\sigma_{r-1} \sigma_{r+1}}$. Since $b_{x} \widetilde{t}_{w^{\prime}}=b_{x} \widetilde{t}_{w^{\prime \prime}} \widetilde{t}_{\sigma_{r+2}}=$ $b_{x} \widetilde{t}_{w^{\prime \prime}}\left(b_{\sigma_{r+2}}-v^{-1}\right)$, we see (as in the proof of Lemma 3.3.1) that for each $y \leq x w^{\prime}$, either $a_{y} \in v^{-1} \mathcal{A}^{-}$or else $a_{y} \in \mathcal{A}^{-}$and $\ell\left(y \sigma_{r+2}\right)<\ell(y)$. In the case where $a_{y} \in$ $v^{-1} \mathcal{A}^{-}$and $a_{y} \neq 0$, we have $a_{y} b_{y} b_{\sigma_{r-1}}=a^{\prime} b_{y^{\prime}}$ with $a^{\prime} \in v^{-1} \mathcal{A}^{-}$by Proposition 3.2.4 (iii) (here, we are using the fact that $\left.\ell\left(y \sigma_{r}\right)<\ell(y)\right)$. But then $a_{y} b_{y} b_{\sigma_{r-1}} b_{\sigma_{r+1}}=$ $a^{\prime} b_{y^{\prime}} b_{\sigma_{r+1}}=a^{\prime \prime} b_{y^{\prime \prime}}$, with $a^{\prime \prime} \in \mathcal{A}^{-}$by Proposition 3.2.4 (i).

Consider now the other case, where $a_{y} \in \mathcal{A}^{-}, a_{y} \neq 0$ and $\ell\left(y \sigma_{r+2}\right)<\ell(y)$. Here, we may write $y$ as a fully commutative reduced product $y=y_{1} \sigma_{r} \sigma_{r+2}$. Notice that $\ell\left(y \sigma_{r-1}\right)>\ell(y)$. If $y \sigma_{r-1}$ is fully commutative, then, since $y \sigma_{r-1}=y_{1} \sigma_{r} \sigma_{r-1} \sigma_{r+2}$ and $\sigma_{r+1} \sigma_{r+2} \neq \sigma_{r+2} \sigma_{r+1}$, we have $a_{y} b_{y} b_{\sigma_{r-1}} b_{\sigma_{r+1}}=a_{y} b_{y \sigma_{r-1}} b_{\sigma_{r+1}}=a^{\prime} b_{y^{\prime}}$ for some $a^{\prime} \in \mathcal{A}^{-}$by Proposition 3.2.4 (iii).

If $y \sigma_{r-1}$ is not fully commutative, then there are two possibilities to consider, depending on whether $r-1$ is greater than or equal to 1. By Proposition 3.2.1 we have, after applying commutations to obtain a suitable reduced expression for $y$ if necessary, either (a) $y=y_{2} \sigma_{r-1} \sigma_{r} \sigma_{r+2}$ (reduced) if $r-1>1$; or (b) $y=$ $y_{3} \sigma_{r} \sigma_{r-1} \sigma_{r} \sigma_{r+2}$ (reduced) if $r-1=1$. The argument for (b) is essentially the same as that for (a), so we treat only (a).

The product $a_{y} b_{y} b_{\sigma_{r-1}} b_{\sigma_{r+1}}$ equals $a_{y} b_{y_{2}} b_{\sigma_{r-1}} b_{\sigma_{r}} b_{\sigma_{r+2}} b_{\sigma_{r-1}} b_{\sigma_{r+1}}$. Since $\sigma_{r+2}$ and $\sigma_{r-1}$ commute, this last expression equals $a_{y} b_{y_{2}} b_{\sigma_{r-1}} b_{\sigma_{r}} b_{\sigma_{r-1}} b_{\sigma_{r+2}} b_{\sigma_{r+1}}=$ $a_{y} b_{y_{2}} b_{\sigma_{r-1}} b_{\sigma_{r+2}} b_{\sigma_{r+1}}$. Now, recall that $y=y_{2} \sigma_{r-1} \sigma_{r} \sigma_{r+2}$ is a fully commutative reduced product. Since $\sigma_{r+2}$ and $\sigma_{r}$ commute, the product $y_{2} \sigma_{r-1} \sigma_{r+2}$ is reduced and fully commutative. It follows that $a_{y} b_{y} b_{\sigma_{r-1}} b_{\sigma_{r+1}}=a_{y} b_{y_{2}} b_{\sigma_{r-1}} b_{\sigma_{r+2}} b_{\sigma_{r+1}}$ equals $a_{y} b_{y_{2} \sigma_{r-1} \sigma_{r+2}} b_{\sigma_{r+1}}$, and this last expression equals $a^{\prime \prime} b_{y^{\prime \prime}}$ for some $a^{\prime \prime} \in \mathcal{A}^{-}$by Proposition 3.2.4 (iii).

Lemma 3.3.8. Let $x \in W_{c}\left(B_{n-1}\right)$ and let $w \in W^{(n)}$. Suppose that there exist $s, s^{\prime} \in S\left(B_{n}\right)$, with $\ell\left(w s s^{\prime}\right)<\ell(w s)<\ell(w)$, such that $x w s s^{\prime}$ is fully commutative but xws is not. Then $b_{x} \widetilde{t}_{w} \in v^{-1} \mathcal{L}_{x w}^{\prime}$. 
Proof. As in the proof of Lemma 3.3.6, we can use Proposition 3.2.1 to divide the argument into two cases: Either (a) $w=\sigma_{n} \sigma_{n-1} \cdots \sigma_{r+1} \sigma_{r} \sigma_{r-1}$, with $2 \leq r<n$ and $\ell\left(x \sigma_{r}\right)<\ell(x)$; or (b) $n \geq 3$ and $w=\sigma_{n} \sigma_{n-1} \cdots \sigma_{2} \sigma_{1} \sigma_{2} \sigma_{3}$ with $\ell\left(x \sigma_{1}\right)<\ell(x)$.

Suppose first that (a) holds. Let $w^{\prime}=w \sigma_{r-1} \sigma_{r} \sigma_{r+1}$ and let $x^{\prime}=x \sigma_{r}$. The product $b_{x} \widetilde{t}_{w}$ equals $b_{x^{\prime}} b_{\sigma_{r}} \widetilde{t}_{w^{\prime}} \widetilde{t}_{\sigma_{r+1}} \widetilde{t}_{\sigma_{r}} \widetilde{t}_{\sigma_{r-1}}$, which in turn can be written as

$$
b_{x^{\prime}} \tilde{t}_{w^{\prime}} b_{\sigma_{r}}\left(b_{\sigma_{r+1}}-v^{-1}\right)\left(b_{\sigma_{r}}-v^{-1}\right)\left(b_{\sigma_{r-1}}-v^{-1}\right) \text {. }
$$

After expanding and then simplifying this last expression, we obtain

$$
b_{x^{\prime}} \widetilde{t}_{w^{\prime}}\left(-v^{-1} b_{\sigma_{r}} b_{\sigma_{r+1}} b_{\sigma_{r-1}}+v^{-2} b_{\sigma_{r}} b_{\sigma_{r+1}}\right)=b_{x} \widetilde{t}_{w^{\prime}}\left(-v^{-1} b_{\sigma_{r+1} \sigma_{r-1}}+v^{-2} b_{\sigma_{r+1}}\right) .
$$

Thus, $b_{x} \widetilde{t}_{w}=-v^{-1} b_{x} \widetilde{t}_{w^{\prime}} b_{\sigma_{r+1} \sigma_{r-1}}+v^{-2} b_{x} \widetilde{t}_{w^{\prime}} b_{\sigma_{r+1}}$. The first of these two terms lies in $v^{-1} \mathcal{L}_{x w}^{\prime}$ by Lemma 3.3.7 and the second lies in $v^{-1} \mathcal{L}_{x w}^{\prime}$ by Lemma 3.3.4.

Suppose now that (b) holds. Let $w^{\prime}=w \sigma_{3} \sigma_{2} \sigma_{1} \sigma_{2} \sigma_{3}$ and let $x^{\prime}=x \sigma_{1}$. The product $b_{x} \widetilde{t}_{w}$ equals $b_{x^{\prime}} b_{\sigma_{1}} \widetilde{t}_{w^{\prime}} \widetilde{t}_{\sigma_{3}} \widetilde{t}_{\sigma_{2}} \widetilde{t}_{\sigma_{1}} \widetilde{t}_{\sigma_{2}} \widetilde{t}_{\sigma_{3}}$, which in turn equals

$$
b_{x^{\prime}} \widetilde{t}_{w^{\prime}} b_{\sigma_{1}}\left(b_{\sigma_{3}}-v^{-1}\right)\left(b_{\sigma_{2}}-v^{-1}\right)\left(b_{\sigma_{1}}-v^{-1}\right)\left(b_{\sigma_{2}}-v^{-1}\right)\left(b_{\sigma_{3}}-v^{-1}\right) .
$$

This last expression expands and then simplifies to

$$
b_{x} \widetilde{t}_{w^{\prime}}\left(v^{-2} b_{\sigma_{3} \sigma_{2} \sigma_{1}}+v^{-2} b_{\sigma_{2} \sigma_{1} \sigma_{3}}-2 v^{-2} b_{\sigma_{3}}-v^{-3} b_{\sigma_{2} \sigma_{1}}+v^{-3}\right) .
$$

The products $-2 v^{-2} b_{x} \widetilde{t}_{w^{\prime}} b_{\sigma_{3}},-v^{-3} b_{x} \widetilde{t}_{w^{\prime}} b_{\sigma_{2} \sigma_{1}}$, and $v^{-3} b_{x} \widetilde{t}_{w^{\prime}}$ belong to $v^{-1} \mathcal{L}_{x w}^{\prime}$ by Lemma 3.3.4. Consider the product $v^{-2} b_{x} \widetilde{t}_{w^{\prime}} b_{\sigma_{3} \sigma_{2} \sigma_{1}}$. If $w^{\prime}=e$, then $n=3$ and $x \sigma_{3} \sigma_{2} \sigma_{1}$ is fully commutative; the latter holds because (1) $x$ is fully commutative and has no reduced expression ending in $\sigma_{2}\left(\right.$ as $\ell\left(x \sigma_{1}\right)<\ell(x)$ and $\sigma_{1}, \sigma_{2}$ do not commute) and (2) the presence of $\sigma_{3}$ precludes the possibility of obtaining a substring of the form $\sigma_{1} \sigma_{2} \sigma_{1} \sigma_{2}$ or $\sigma_{2} \sigma_{1} \sigma_{2} \sigma_{1}$ through commutation moves. Hence, $v^{-2} b_{x} \widetilde{t}_{w^{\prime}} b_{\sigma_{3} \sigma_{2} \sigma_{1}}=v^{-2} b_{x \sigma_{3} \sigma_{2} \sigma_{1}} \in v^{-1} \mathcal{L}_{x w}^{\prime}$. If $w^{\prime} \neq e$, then $v^{-2} b_{x} \widetilde{t}_{w^{\prime}} b_{\sigma_{3} \sigma_{2} \sigma_{1}} \in \mathcal{L}_{x w}^{\prime}$ by Lemma 3.3 .5 (i) (taking $s=\sigma_{4}$ ). Finally, we have $b_{x} \widetilde{t}_{w^{\prime}} b_{\sigma_{2} \sigma_{1}} \in \mathcal{L}_{x w^{\prime} \sigma_{3} \sigma_{2} \sigma_{1}}^{\prime}$ by Lemma 3.3 .5 (ii) (taking $s=\sigma_{1}$ ), which, by Proposition 3.2.4 (i), shows that $v^{-2} b_{x} \widetilde{t}_{w^{\prime}} b_{\sigma_{2} \sigma_{1} \sigma_{3}} \in v^{-1} \mathcal{L}_{x w}^{\prime}$. 
Lemma 3.3.9. Let $x \in W_{c}\left(B_{n-1}\right)$ and let $w \in W^{(n)}$. Suppose that $x w$ is not fully commutative. Then $b_{x} \widetilde{t}_{w} \in v^{-1} \mathcal{L}_{x w}^{\prime}$.

Proof. We proceed by induction on $\ell(w)$. The lemma is (vacuously) true for $\ell(w)<$ 2 , since $x w$ is fully commutative for such $w \in W^{(n)}$. Suppose that $\ell(w) \geq 2$. Let $s, s^{\prime}$ be (the) Coxeter generators that satisfy $\ell\left(w s s^{\prime}\right)<\ell(w s)<\ell(w)$. If $x w s s^{\prime}$ is not fully commutative, then neither is $x w s$; by the inductive hypothesis, $b_{x} \widetilde{t}_{w s s^{\prime}} \in v^{-1} \mathcal{L}_{x w s s^{\prime}}^{\prime}$ and $b_{x} \widetilde{t}_{w s} \in v^{-1} \mathcal{L}_{x w s}^{\prime}$. But then $b_{x} \widetilde{t}_{w} \in v^{-1} \mathcal{L}_{x w}^{\prime}$ by Lemma 3.3.1 (taking $k=1$ ).

On the other hand, suppose that $x w s s^{\prime}$ is fully commutative. If $x w s$ is not fully commutative, then Lemma 3.3 .8 gives us $b_{x} \widetilde{t}_{w} \in v^{-1} \mathcal{L}_{x w}^{\prime}$. Finally, if $x w s$ is fully commutative, then Lemma 3.3.6 applies.

The inductive step is complete.

Proposition 3.3.10. We have $\widetilde{t}_{w} \in v^{-1} \mathcal{L}_{w}^{\prime}$ for all $w \in W\left(B_{n}\right) \backslash W_{c}\left(B_{n}\right)$.

Proof. Let $w \in W\left(B_{n}\right) \backslash W_{c}\left(B_{n}\right)$. Write $w=w_{1} w_{2} \cdots w_{n}$, where each $w_{i} \in W^{(i)}$. There exists a unique integer $r>1$ such that $w_{1} w_{2} \cdots w_{r-1}$ is fully commutative and $w_{1} w_{2} \cdots w_{r}$ is not. Let $y=w_{1} w_{2} \cdots w_{r-1}$. Given the nature of the representative $w_{r}$, it follows from Proposition 3.2.1 that $y=y^{\prime} s$ (reduced), where $s \in S\left(B_{r-1}\right)$ is such that $s w_{r}$ is not fully commutative. By Proposition 3.3.3, we have $\widetilde{t}_{y^{\prime}} \in \mathcal{L}_{y^{\prime}}^{\prime}$ and $\widetilde{t}_{y} \in \mathcal{L}_{y}^{\prime}$. Combining this with the equality $\widetilde{t}_{y}=\widetilde{t}_{y^{\prime}}\left(b_{s}-v^{-1}\right)$, we find that if $\widetilde{t}_{y}$ is written as a linear combination of monomial basis elements $b_{x}(x \leq y)$, then for each $x$, either the coefficient of $b_{x}$ lies in $v^{-1} \mathcal{A}^{-}$, or the coefficient lies in $\mathcal{A}^{-}$and $\ell(x s)<\ell(x)$.

Thus, $\widetilde{t}_{y w_{r}}=\widetilde{t}_{y} \widetilde{t}_{w_{r}}$ equals a sum of terms of the form $a_{x} b_{x} \widetilde{t}_{w_{r}}(x \leq y)$, where for each $x$, either $a_{x} \in v^{-1} \mathcal{A}^{-}$, or else $a_{x} \in \mathcal{A}^{-}$and $x w_{r}$ is not fully commutative. Now, by Lemma 3.3.2, we have $b_{x} \widetilde{t}_{w_{r}} \in \mathcal{L}_{x w_{r}}^{\prime}$. Therefore, in the case where $a_{x} \in v^{-1} \mathcal{A}^{-}$, we have $a_{x} b_{x} \widetilde{t}_{w_{r}} \in v^{-1} \mathcal{L}_{x w_{r}}^{\prime}$. In the case where $a_{x} \in \mathcal{A}^{-}$and $x w_{r} \notin W_{c}$, Lemma 3.3.9 gives us $a_{x} b_{x} \widetilde{t}_{w_{r}} \in v^{-1} \mathcal{L}_{x w_{r}}^{\prime}$. We have so far shown that $\widetilde{t}_{y w_{r}} \in v^{-1} \mathcal{L}_{y w_{r}}^{\prime}$.

The product $\widetilde{t}_{y w_{r}} \widetilde{t}_{w_{r+1}}$ must then lie in $v^{-1} \mathcal{L}_{y w_{r} w_{r+1}}^{\prime}$. To see this, expand $\widetilde{t}_{y w_{r}}$ 
into a sum of terms $a_{z}^{\prime} b_{z}\left(z \leq y w_{r}\right)$, where each $a_{z}^{\prime} \in v^{-1} \mathcal{A}^{-}$. Then $\widetilde{t}_{y w_{r}} \widetilde{t}_{w_{r+1}}$ equals a sum of terms $a_{z}^{\prime} b_{z} \widetilde{t}_{w_{r+1}}$, each of which must lie in $v^{-1} \mathcal{L}_{y w_{r} w_{r+1}}^{\prime}$ by Lemma 3.3.2 (here, $r+1$ is playing the role of $n$ in the lemma). By iterating this argument, we are able to conclude that $\widetilde{t}_{w}=\widetilde{t}_{y w_{r}} \widetilde{t}_{w_{r+1}} \cdots \widetilde{t}_{w_{n}} \in v^{-1} \mathcal{L}_{w}^{\prime}$, as desired.

\subsection{Proof of Theorem 3.1.1.}

We are now in a position to give a proof that the conditions of Theorem 2.2.3 are satisfied when the underlying Coxeter graph is of type $B_{n}$.

Proof of Theorem 3.1.1. We shall verify condition (v) of Theorem 2.2.3. It is required to prove that for each $w \in W\left(B_{n}\right) \backslash W_{c}\left(B_{n}\right)$, the element $\theta\left(\widetilde{T}_{w}\right)=\widetilde{t}_{w}$ lies in $v^{-1} \mathcal{L}$. Proposition 3.3 .10 gives $\widetilde{t}_{w} \in v^{-1} \mathcal{L}_{w}^{\prime}$ for such $w$. Thus, the theorem will follow if we can show that $\mathcal{L}_{w}^{\prime}=\mathcal{L}_{w}$.

Fix an arbitrary $w$. Proposition 3.3.3 gives $\mathcal{L}_{w}^{\prime} \supseteq \mathcal{L}_{w}$. Let $x \in W_{c}$ satisfy $x \leq w$. By Proposition 3.3.3, we may write $\widetilde{t}_{x}=\sum a_{y} b_{y}$, where for all $y$ we have $y \leq x$, $y \in W_{c}$ and $a_{y} \in \mathcal{A}^{-}$. Moreover, given any reduced expression $s_{1} s_{2} \cdots s_{m}$ for $x$, we have

$$
\widetilde{t}_{x}=\widetilde{t}_{s_{1}} \widetilde{t}_{s_{2}} \cdots \widetilde{t}_{s_{m}}=\left(b_{s_{1}}-v^{-1}\right)\left(b_{s_{2}}-v^{-1}\right) \cdots\left(b_{s_{m}}-v^{-1}\right),
$$

from which we can see that $a_{x}=1$. It now follows by a straightforward induction on the Bruhat-Chevalley order that any $b_{x}$ with $x \leq w$ can be written as an $\mathcal{A}^{-}$linear combination of basis elements $\widetilde{t}_{y}$ with $y \leq x$. That is, we have $\mathcal{L}_{w}^{\prime} \subseteq \mathcal{L}_{w}$, as well.

Remark 3.4.1. Another possible approach to proving Theorem 3.1 .1 would be to use the combinatorial classification of cells achieved by Garfinkle in $[\mathbf{5}, \mathbf{6}, \mathbf{7}]$.

We conclude with a discussion of an application: it is possible as a consequence of Theorem 3.1.1 to describe very explicitly the structure of the fully commutative left, right and two-sided cells in type $B$. By [12, Theorem 2.2.1], the canonical basis for $\mathcal{T} \mathcal{L}\left(B_{n}\right)$ is the image under $\theta$ of the set $\left\{C_{w}^{\prime}: w \in W_{c}\left(B_{n}\right)\right\}$, and by condition (iii) of Theorem 2.2.3, all other $C_{w}^{\prime}$ are mapped to zero. The diagram calculus for 
the canonical basis of $\mathcal{T L}\left(B_{n}\right)$ given in [10, Theorem 2.2.5] can now be used to describe the various kinds of fully commutative Kazhdan-Lusztig cells.

By [16, Theorem 1.10], each left (respectively, right) cell in a crystallographic Coxeter group $W$, such as $W\left(B_{n}\right)$, contains a unique distinguished involution $d$. In type $A$, any left cell and right cell from the same two-sided cell intersect in a single element. Our approach can be used to describe the situation in type $B$ in an elementary way.

Let $n \geq 2$, and let $W^{\prime} \cong W\left(A_{n-1}\right)$ be the parabolic subgroup of $W\left(B_{n}\right)$ obtained by omitting the first generator. Let $d, d^{\prime} \in W\left(B_{n}\right)$ be distinguished involutions in the same fully commutative two-sided cell, $I_{T}$. Let $I_{R}$ be the right cell containing $d$, and let $I_{L}$ be the left cell containing $d^{\prime}$. Let $k=\left|I_{R} \cap I_{L}\right|$. Then we have $k=1$ if exactly one of the elements $d, d^{\prime}$ lies in $W^{\prime}$, or if $I_{T} \subseteq W^{\prime}$, or if $I_{T} \cap W^{\prime}=\emptyset$. Otherwise, we have $k=2$.

\section{REFERENCES}

[1] F. du Cloux, Coxeter Version 1.01, Université de Lyon, France, 1991.

[2] C.K. Fan, A Hecke algebra quotient and properties of commutative elements of a Weyl group, Ph.D. thesis, M.I.T., 1995.

[3] C.K. Fan and R.M. Green, Monomials and Temperley-Lieb algebras, J. Algebra 190 (1997), 498-517.

[4] C.K. Fan and J.R. Stembridge, Nilpotent orbits and commutative elements, J. Algebra 196 (1997), 490-498.

[5] D. Garfinkle, On the classification of primitive ideals for complex classical Lie algebras, I, Compositio Math. 75 (1990), 135-169.

[6] On the classification of primitive ideals for complex classical Lie algebras, II, Compositio Math. 81 (1992), 307-336.

[7] On the classification of primitive ideals for complex classical Lie algebras, III, Compositio Math. 88 (1993), 187-234.

[8] J.J. Graham, Modular representations of Hecke algebras and related algebras, Ph.D. thesis, University of Sydney, 1995.

[9] R.M. Green, Generalized Temperley-Lieb algebras and decorated tangles, J. Knot Theory Ramifications 7 (1998), 155-171.

[10] Decorated tangles and canonical bases (preprint).

[11] R.M. Green and J. Losonczy, Canonical bases for Hecke algebra quotients, Math. Res. Lett. 6 (1999), 213-222.

[12] A projection property for Kazhdan-Lusztig bases, Internat. Math. Res. Notices 1 (2000), 23-34.

[13] J.E. Humphreys, Reflection Groups and Coxeter Groups, Cambridge University Press, Cambridge, 1990.

[14] D. Kazhdan and G. Lusztig, Representations of Coxeter groups and Hecke algebras, Invent. Math. 53 (1979), 165-184. 
[15] J. Losonczy, The Kazhdan-Lusztig basis and the Temperley-Lieb quotient in type D, J. Algebra 233 (2000), 1-15.

[16] G. Lusztig, Cells in affine Weyl groups, II, J. Algebra 109 (1987), 536-548.

[17] J.R. Stembridge, On the fully commutative elements of Coxeter groups, J. Algebraic Combin. 5 (1996), 353-385. 